\title{
PASTEURELLA MULTOCIDA TENOSYNOVITIS OF THE FLEXOR POLLICIS LONGUS CAUSED BY A RABBIT BONE WOUND
}

Blasco, Alejandro; Cuñat, Borja; Baixauli, Emilio;Amaya, José

A 29-year-old man was admitted to our emergency department with a painful thumb, feverredness, and swelling and limited function. Five days before he was wounded with a rabbitbone. He was given amoxicillin-clavulanate with a lack of improvement. He was admitted forhospitalization and an ultrasound confirmed tenosynovitis as well as subcutaneous edema, sosurgical debridement was performed. Cultures were positive to Pasteurella multocida.Hospital stay was 9 days, and he continued oral antibiotic for 10 days after discharge. Fourmonths postoperatively, the patient had complete function and didn't show evidence ofrecurrence.

To conclude, rapidly developing cellulitis, tenosynovitis, fever and drainage fromhand wounds after a cat or dog bites should suggest Pasteurella multocida infection. However, P. multocida tenosynovitis can be also produced after a rabbit bone wound.Absence of response after 24-48 of antibiotic treatment, especially if cellulitis has progressedto tenosynovitis, is an indication for surgery. 


\section{BACKGROUND}

Pasteurella multocida has been previously considered as the major cause of infections following domestic animal bites, especially after dog or cat bite.[1] However, there are few publications about hand infections and tenosynovitis associated with rabbit wound. We obtained a total of 10 articles in an advanced Pubmed search including the terms pasteurellamultocida AND tenosynovitis in humans. We excluded 4 articles (paediatrics, other languages, or other animals) and finally we obtained 6 articles related directly with Pasteurella and tenosynovitis in humans. We obtained 46 articles in an advanced Pubmed search in humans including MeSH terms infection, pasteurella and hand. We couldn't find new articles of pasteurella infection with rabbit contact after reading titles and summaries and papers are commonly focused on cat and dogs bites and scratches. Finally, to perform this publication we added 9 more articles related with our issue. For that reason we are strongly convinced that it was a rare but elucidating case.

\section{CASE PRESENTATION}

A 29-year-old man from the North African Maghreb region was admitted to our emergency department with a painful right thumb, a fever of $38,8^{\circ} \mathrm{C}$ with redness, and swelling and limited function. He was otherwise healthy; no history of renal failure or hepatic cirrhosis, he didn't take corticosteroids or any other medication. He referred that five days before he was wounded with a rabbit bone, while he was chopping a rabbit for cooking (he bought the meat with the offal discarded). He started feeling more pain the next day and he visited his general practitioner 4 days after de initial wound and he was given the tetanus vaccine and he was given a prescription of one amoxicillin-clavulanate tablet $875 \mathrm{mg} / 125 \mathrm{mg}$ every 8 hours and a fusidic acid cream. He claimed a lack of improvement with the medication prescribed. His vital signs were otherwise normal and the physical examination revealed a volar thumb fluctuating erythema suggesting a purulent collection.

\section{INVESTIGATIONS}

An ultrasound performed at the emergency department reported a suspicion of flexor pollicis longus, flexors of the digits and flexor carpi radialis tenosynovitis as well as subcutaneous edema. No evidence of foreign body was reported. Blood test showed a normal blood count with left deviation of neutrophils (leukocytes were $10300 / \mu \mathrm{L}$ and neutrophils were $7760 / \mu \mathrm{L}$ ), elevated C Reactive Protein $125,7 \mathrm{mg} / \mathrm{L}$, erythrocyte sedimentation rate $20 \mathrm{~mm} / \mathrm{h}$ and elevated fybrinogen $675 \mathrm{mg} / \mathrm{dL}$.

\section{DIFFERENTIAL DIAGNOSIS}

With these findings, the diagnostic suspicion of tenosynovitis of the flexor pollicis longus caused by a rabbit bone wound was made.

\section{TREATMENT}

He was admitted for hospitalization with intravenous amoxicillin-clavulanate treatment. With the diagnostic suspicion of tenosynovitis, six days after the initial wound, a surgical debridement was performed (Figure 1). A volar approach of the thumb was iniciated and evidence of purulent material appeared in the synovial sheath of flexor pollicis longus so the surgical incision was prolonged proximally. Following the carpal tunnel, and preserving the motor branch of the median nerve, surgical incision was extended to the flexor carpi radialis sheath (attending the findings of the previous ultrasound examination). A release of de $A 1$ polley of the thumb was 
performed, while multiple samples of purulent material were sent for microscopy, culture and sensitivity tests. An exhaustive cleaning was carried out with 4 liters of physiological saline and skin was closed with monofilament sutures. A plastersplintwasapplied to thepatient.

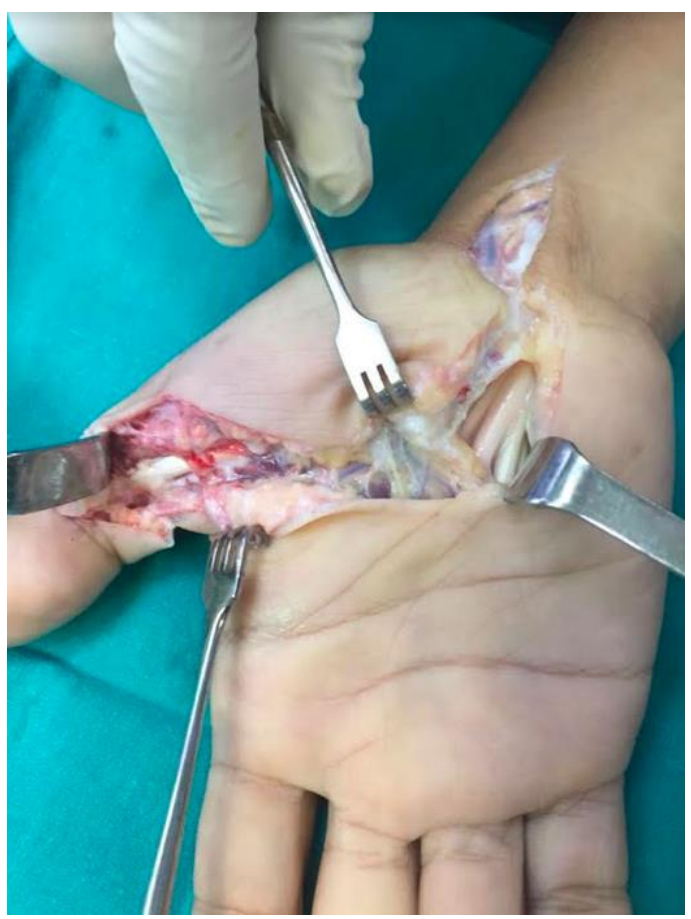

Figure 1. A volar approach of the thumb was initiated and evidence of purulent material appeared in the synovial sheath of flexor pollicis longus so the surgical incision was prolonged proximally. Following the carpal tunnel, and preserving the motor branch of the median nerve, surgical incision was extended to the flexor carpi radialis sheath (attending the findings of the previous ultrasound examination). A release of de Th1 polley of the thumb was performed.

\section{OUTCOME AND FOLLOW-UP}

Cultures were positive to Pasteurella multocida sensitive to amoxicillin and clavulanate, so the intravenous treatment was continued. Hospital stay was 9 days, and he continued oral antibiotic for 10 days after discharge. At the time of discharge, the wound showed no signs of infection. The patient had a normal body temperature $\left(36,7^{\circ} \mathrm{C}\right)$ as well as normal vital signs (129/79mmHg blood pressure and $72 \mathrm{bpm})$. Blood tests reported that the c-reactive protein had normalized to $4,4 \mathrm{mg} / \mathrm{L}$, leukocytes were $5940 / \mu \mathrm{L}$. A follow-up was carried out in outpatient consultations and the patient followed a targeted physiotherapy treatment getting a normal range of mobility. Two months after debridement, a blood test was performed and both the $c$ reactive protein $(0,8 \mathrm{mg} / \mathrm{L})$, and leukocytes $(5530 / \mu \mathrm{L})$ remained within normal ranges. Four months postoperatively mobility became normal, he was asymptomatic, without new purulent collections, so finally, the patient was discharged.

\section{DISCUSSION}

Clinical presentation. To our kwnoledge, this is the first tenosynovitis described after a rabbit bone wound. Pasteurella multocida wound infections in humans most often result from direct inoculation via bites including cats bites, cat scratches, or dog bites that usually cause cellulitis or abscesses.[2] However, P. multocida infections have been also described after contact with opposum, rat, lion, rabbit, pig, wolf, turkeys and horse.[2,3] More severe infections, which include bursitis, septic arthritis, osteomyelitis, pneumonia, meningitis, peritonitis, and bacteremia, are rare but more frequently seen in immunocompromised patients. [2,4] P. multocida acute infection of a total knee and shoulder arthroplasty after a cat and dogs scratches or bites have also been published.[5-8] 
Tenosynovitis of the hand. When to suspect P. multocida? Our patient took 5 days after visiting general practitioner; with a painful right thumb, a fever of $38,8{ }^{\circ} \mathrm{C}$ with redness, and swelling and limited function. Superficial skin and soft tissue infections constitute most $P$. multocidainfections in humans. They are characterized by the rapid appearance of erythema, warmth, tenderness, and purulent drainage. $[1,2,4]$ Boisvert describes a Pasteurella lepiseptica infection (antique name for Pasteurella multocida from rabbits) after a rabbit bite on the right index finger that progressed to the dorsum of the hand and the forearm and required abscess drainage 28 days after the bite.[9] Arons reports a total of 55 patients positive for $P$. multocida following domestic cat or dog bite injuries (none after rabbit wound).[1]

Weber, in a series of 23 patients with $P$. multocida infection reports 16 infections involving the upper extremity $(69,56 \%)$, but most of them where cellulitis and they only reported 1 tenosynovitis (after dog bite).[2] Monsey reported a tenosynovitis after cat scratch that resolved with antibiotic treatment (no surgery required).[10] García-Triana reported 2 cases of Pasteurella multocida tenosynovitis of the hand after cat scratch and bite that required surgical debridement and irrigation plus antibiotic treatment.(4) In a series of 108 pyogenic tenosynovitis that required débridement, Müller et al reported that the most common cause was Staphylococcus aureus (27\%), followed by Pasteurella multocida (7\%) and Streptococcus pyogenes (6\%).[11] Other authors have elucidated indeed that acute suppurative tenosynovitis is more frequently caused by Staphylococcus aureus or Streptococcus pyogenes but the contact with cats or dogs should suggest us a $P$. multocidainfection because it is the most common pathogen after cat and dog bites.[4,12,13] In comparison to staphylococci or streptococci, the isolation of Pasteurellaspecies is associated with a shorter latency period.[12] Patient idiosyncrasy and epidemiologic exposures may give us clues to uncommon pathogens that occasionally cause tenosynovitis, such as Neisseria gonorrhoeae, Pasteurella multocida, atypical mycobacteria, fungi, and protothecosis.[14]So the fast onset of cellulitis, lymphangitis, and serosanguineous or purulent drainage from hand wounds 12 to 24 hours after cat or dog bites should suggest Pasteurella multocidainfection.[1]

Step by step diagnosis. The four classic signs of flexor tenosynovitis are: 1) tenderness over the course of the flexor sheath, 2) symmetric enlargement of the affected finger, 3) slightly flexed finger at rest, and 4) pain along the tendon sheath with passive extension of the finger.[14] Fever, leukocyte count with left shift and C-reactive protein are usually elevated but could be normal.[14] Plain $\mathrm{X}$-rays studies are recommendable to rule out fractures and metallic foreign bodies.[14]Ultrasound is the test of choice to confirm tenosynovitis and 
may guide diagnostic needle aspiration.[4,14] MRI and CT might also be useful for determining extent of infection.[14]

Microbiology. Culture of the organism is not difficult if the laboratory is alerted to the origin of the specimen and the suspected organisms.[4] Definitive diagnosis is made by Gram stain and culture of synovial sheath fluid, by aspiration or at the time of surgical intervention.[14] P. multocida is a small gram-negative, fermentative and nonmotile coccobacillus, part of the normal oral flora of cats and dogs.[1,2,5,8] It can grow on normal media but grows better on blood or serum-containing media.[7] Pasteurella multocida has positive reactions for catalase, oxidase and nitrate reduction but negative reaction for urease.[2]

Treatment. Should we operate it? When? In our case, even after correct antibiotic treatment, tenosynovitis progressed.Early diagnosis and treatment are crucial for preventing devastating complications such as tendon necrosis and contamination of other compartments in the hand or adjacent joints.[4]Prophylactic antibiotics are not recommended in simple hand lacerations.[1] For simple puncture wounds leading to tenosynovitis, appropriate parenteral staphylococcal and streptococcal therapy may be sufficient.[2,14] Primarily, when treating bite wounds with secondary tenosynovitis, broader spectrum antibiotics with additional activity against gram-negative and anaerobic bacteria are indicated, as we did. $[14,15]$ The most accurate oral antibiotics for Pasteurella multocida infections are: penicillin $\mathrm{V}$, amoxicillin, amoxicillin-clavulanate, cefuroxime, tetracycline, and ciprofloxacin.[4]

Surgical intervention may be necessary if antibiotics alone are not effective after 24 to 48 hours of therapy, or if the patient presents after the first 2 days of symptoms; both assertion took place in our case.[14] While surgery may not be as urgent in cases of extensor tenosynovitis, a close monitoring is also then mandatory because they can also be devastating.[14,15] In our case there was no need but persistence or secondary worsening of infection may be a cause for of subsequent debridement.[11]

\section{LEARNING POINTS/TAKE HOME}

\section{MESSAGES}

- A fast onset of cellulitis, tenosynovitis, fever and drainage from hand wounds after a cat or dog bite should suggest Pasteurella multocidainfection. However, P. multocida tenosynovitis can be also produced after a rabbit bone wound.

- Pasteurella multocidainfection usually responds to antibiotherapy (penicillin $\mathrm{V}$, amoxicillin, amoxicillin-clavulanate, cefuroxime, tetracycline, and ciprofloxacin). 
- One has to consider that a lack of response after 24-48 of antibiotic treatment, especially if cellulitis has progressed to tenosynovitis, is an indication for surgery (culture and sensitivity tests and debridement and irrigation).

\section{REFERENCES}

1. Arons MS, Fernando L, Polayes IM. Pasteurella multocida--the major cause of hand infections following domestic animal bites. J Hand Surg. 1982 Jan;7(1):47-52.

2. Weber DJ, Wolfson JS, Swartz MN, Hooper DC. Pasteurella multocida infections. Report of 34 cases and review of the literature. Medicine (Baltimore). 1984 May;63(3):133-54.

3. Silberfein EJ, Lin $\mathrm{PH}$, Bush RL, Zhou W, Lumsden AB. Aortic endograft infection due to Pasteurella multocida following a rabbit bite. J Vasc Surg. 2006 Feb;43(2):393-5.

4. García Triana $M$, Fernández Echevarria MA, Alvaro RL, Sagredo PS, Parra Blanco JA. Pasteurella multocida tenosynovitis of the hand: sonographic findings. J Clin Ultrasound JCU. 2003 Apr;31(3):159-62.

5. Heydemann J, Heydemann JS, Antony $S$. Acute infection of a total knee arthroplasty caused by Pasteurella multocida: a case report and a comprehensive review of the literature in the last 10 years. Int J Infect Dis IJID Off Publ Int Soc Infect Dis. 2010 Sep;14 Suppl 3:e242-245.
6. Miranda I, Angulo M, Amaya JV. [Acute total knee replacement infection after a cat bite and scratch: a clinical case and review of the literature]. Rev Espanola Cirugia Ortop Traumatol. 2013 Aug;57(4):300-5.

7. Ding DY, Orengo A, Alaia MJ, Zuckerman JD. Pasteurella multocida infection in a primary shoulder arthroplasty after cat scratch: case report and review of literature. J Shoulder Elbow Surg. 2015 Jun;24(6):e159-163.

8. Honnorat $E$, Seng $P$, Savini $H$, Pinelli P-O, Simon F, Stein A. Prosthetic joint infection caused by Pasteurella multocida: a case series and review of literature. BMC Infect Dis. 2016 20;16(1):435.

9. Boisvert PL, Fousek MD. Human infection with pasteurella leptiseptica following a rabbit bite. JAMA. 1941 Apr;116(17):1902-3.

10. Monsey R, Mogan J. Pasteurella flexor tenosynovitis. Orthopedics. 1993 May;16(5):620-2.

11. Müller $\mathrm{CT}$, Uçkay I, Erba P, Lipsky BA, Hoffmeyer P, Beaulieu J-Y. Septic Tenosynovitis of the Hand: Factors Predicting Need for Subsequent Débridement. Plast Reconstr Surg. 2015 Sep;136(3):338e-343e.

12. Abrahamian FM, Goldstein EJC. Microbiology of animal bite wound infections. Clin Microbiol Rev. 2011 Apr;24(2):231-46. 
13. Talan DA, Citron DM, Abrahamian FM, Moran GJ, Goldstein EJ. Bacteriologic analysis of infected dog and cat bites. Emergency Medicine Animal Bite Infection Study Group. N Engl J Med. 1999 Jan 14;340(2):85-92.

14. Small LN, Ross JJ. Suppurative tenosynovitis and septic bursitis. Infect Dis Clin North Am. 2005 Dec;19(4):9911005 , xi.

15. Vives-Barquiel $M$, BallesterosBetancourt JR, García-Tarriño R, Fernández-Valencia J, Llusá M, García B, et al. Salvage of upper extremity in an aggressive case of Pasteurella multocida infection. Am J Emerg Med. 2016 Dec;34(12):2470.e1-2470.e3. 